\title{
BMJ Open Immunogenicity and safety of early vaccination with two doses of a combined measles-mumps-rubella- varicella vaccine in healthy Indian children from 9 months of age: a phase III, randomised, non-inferiority trial
}

\author{
Sanjay Lalwani, ${ }^{1}$ Sukanta Chatterjee, ${ }^{2}$ Sundaram Balasubramanian, ${ }^{3}$ \\ Ashish Bavdekar, ${ }^{4}$ Shailesh Mehta, ${ }^{5}$ Sanjoy Datta, ${ }^{6}$ Michael Povey, ${ }^{6}$ \\ Ouzama Henry ${ }^{7}$
}

To cite: Lalwani S,

Chatterjee S,

Balasubramanian $\mathrm{S}$, et al. Immunogenicity and safety of early vaccination with two doses of a combined measles-mumps-rubellavaricella vaccine in healthy Indian children from 9 months of age: a phase III, randomised, non-inferiority trial. BMJ Open 2015;5: e007202. doi:10.1136/ bmjopen-2014-007202

- Prepublication history and additional material is available. To view please visit the journal (http://dx.doi.org/ 10.1136/bmjopen-2014007202).

Received 14 November 2014 Revised 21 May 2015 Accepted 22 May 2015

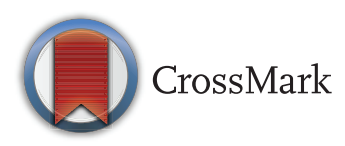

For numbered affiliations see end of article.

Correspondence to Dr Sanjay Lalwani; sanjaylalwani2007@ rediffmail.com

\section{ABSTRACT}

Objective: This study (NCT00969436) compared the immunogenicity and safety of measles-mumps-rubella (MMR) followed by MMR+varicella (V) vaccines to (1) 2 doses of combined MMRV and (2) MMR followed by MMRV, in Indian children.

Design: Phase III, open, randomised, non-inferiority study.

Setting: 6 tertiary care hospitals located in India.

Participants: Healthy participants aged 9-10 months not previously vaccinated against/exposed to measles, mumps, rubella and varicella or without a history of these diseases.

Interventions: Participants were randomised (2:2:1) to receive 2 doses of either MMRV (MMRV/MMRV group) or MMR followed by MMRV (MMR/MMRV group) or MMR followed by MMR $+\mathrm{V}$ (MMR/MMR+V, control group) at 9 and 15 months of age. Antibody titres against measles, mumps and rubella were measured using ELISA and against varicella using an immunofluorescence assay.

Main outcome measures: To demonstrate noninferiority of the 2 vaccination regimens versus the control in terms of seroconversion rates, defined as a group difference with a lower bound of the $95 \% \mathrm{Cl}$ $>-10 \%$ for each antigen, 43 days postdose 2. Parents/ guardians recorded solicited local and general symptoms for a 4-day and 43-day period after each vaccine dose, respectively.

Results: Seroconversion rates postdose 1 ranged from $87.5 \%$ to $93.2 \%$ for measles, $83.3 \%$ to $86.1 \%$ for mumps and $98.7 \%$ to $100 \%$ for rubella across the 3 vaccine groups. The seroconversion rates postdose 2 were $100 \%$ for measles, mumps and rubella and at least $95.8 \%$ for varicella across the 3 vaccine groups. Non-inferiority of MMRV/MMRV and MMR/MMRV to MMR/MMR+V was achieved for all antigens, 43 days postdose 2 . The 3 vaccination regimens were generally well tolerated in terms of solicited local and general symptoms.

\section{Strengths and limitations of this study}

This Indian study provides data for the first time on:

- A combined measles-mumps-rubella-varicella (MMRV) vaccine in a highly endemic measles setting.

- MMRV administered to children at 9 months of age, which aligns with the expanded programme of immunisation schedule of measles vaccine administered at this age.

- Prevaccination serostatus that offers epidemiological indicators on the early disease burden for measles, mumps, rubella and varicella.

- The six tertiary care centres where the study was conducted are not representative of the entire Indian population.

- There was investigator bias while reporting adverse events due to the open design of the study.

- There were no adjustments made for confounding factors (eg, centres) in the analysis.

Conclusions: The immune responses elicited by the MMRV/MMRV and MMR/MMRV vaccination regimens were non-inferior to those elicited by the MMR/MMR+V regimen for all antigens. The 3 vaccination schedules also exhibited an acceptable safety profile in Indian children.

Trial registration number: NCT00969436.

\section{INTRODUCTION}

Measles, mumps, rubella and varicella are highly infectious vaccine-preventable childhood diseases that continue to pose a 
significant public health problem in India and beyond. ${ }^{1-4}$ In 2010, global measles mortality was estimated at 139000 (71 200-447 800) deaths, $47 \%$ of which was estimated to have occurred in India. ${ }^{5}$ In 2011, large measles outbreaks were reported in India (29 339 cases), Pakistan (4386 cases), Nigeria (18 843 cases) and other countries. ${ }^{6}$ Although measles elimination was declared in the USA in 2000 , the importation of the disease led to the highest number of cases in 2011 (220 cases) since 1996, while 159 cases were reported in 2013 by 16 states. ${ }^{7}$ In the European Union, the Dutch authorities reported 1540 measles cases since May 2013, and in Germany the reported number of cases is nearly 10 times higher than the total cases in 2012. ${ }^{8}$ A large number of confirmed cases of measles was also reported in England and Wales between 2012 and 2013, respectively. ${ }^{9} 10$ Thus, even developed settings may be prone to epidemics if coverage wanes. ${ }^{7-10}$

A dramatic decrease in the worldwide mumps disease burden has been observed since the implementation of large-scale immunisation in $1967 .^{2}$ However, the true incidence in India is difficult to ascertain due to limited baseline epidemiological data. ${ }^{11}$

A study conducted in 2006 revealed that $82.2 \%$ of children aged between 1 and 5 years, and $13.5 \%$ aged between 10 and 15 years are susceptible to rubella in the state of Tamil Nadu in southern India. ${ }^{12}$ Although congenital rubella syndrome (CRS) has been reported in most parts of India, no measures have been undertaken to control this crippling disease, and presently there are limited reliable data on CRS in India. ${ }^{3}$

Epidemiological data on varicella-zoster virus are also scarce in India as chickenpox was not a notifiable disease in India until $2005,{ }^{14}$ and owing to the locally perceived self-limiting and relatively less severe nature, the disease is under-reported.

Globally, routine and effective vaccination has been identified as a critical approach towards achieving high and sustained vaccination coverage rates and to strategically deal with the burden of these four diseases. ${ }^{15}$ Consequently, the Indian Academy of Pediatrics (IAP) has recommended the inclusion of a combined measles-mumps-rubella (MMR) vaccine in the national immunisation schedule to provide protection against CRS and also to reduce the disease burden of measles and mumps. ${ }^{16}$ In countries with ongoing measles transmission, the WHO recommends a first dose of measles vaccine at 9 months of age to afford early protection and a second dose at 15-18 months with a minimum interval of 1 month between the two doses. ${ }^{1}$ In India, the observed high morbidity and mortality due to measles have necessitated the administration of the measles vaccine at 9 months of age (by which time most children will have lost their maternal antibodies to measles) ${ }^{17}$ followed by MMR at 15 months of age. ${ }^{18}$ The IAP also recommends two doses of a varicella vaccine, with the first dose administered at 15 months of age. ${ }^{18}$ The second dose may be administered 3 months after the first, but is usually given at $4-6$ years. ${ }^{18}$ There is increasing global evidence in many settings that the high economic burden of varicella would be beneficially alleviated with the inclusion of varicella vaccine. ${ }^{19-21}$

GlaxoSmithKline's (GSK) MMR and varicella vaccines are available in over 100 countries $^{22}$ and 80 countries, ${ }^{23}$ respectively. These vaccines are currently not included in the Indian national (government-provided) immunisation programme; however, they are available via private practitioners. On the basis of commercially available formulations, a combined MMR-varicella (MMRV) vaccine has been developed to realise the benefits of vaccination against measles, mumps and rubella, as well as to facilitate the potential inclusion of varicella into national immunisation programmes. $^{24-27}$ The new vaccine is as immunogenic as separate MMR and varicella vaccinations. ${ }^{28-30}$

This study evaluated the non-inferiority of two different vaccination regimens of the new MMRV vaccine to the control regimen of separate injections when the vaccines were administered at 9 and 15 months of age to healthy Indian children.

\section{METHODS}

\section{Study design and participants}

This phase IIIb, open, randomised, controlled study (NCT00969436) was conducted at six tertiary care centres (see online supplementary table) in India between November 2009 and February 2011. The open nature of the study implied that both the investigators and the parents/guardians were aware of the treatment; however, the laboratory personnel generally were unaware of the treatment allocation. Healthy participants aged 9-10 months were randomised (2:2:1) to receive either two doses of the MMRV vaccine (MMRV/MMRV group) or MMR followed by MMRV (MMR/MMRV group), or MMR followed by MMR+V (MMR/MMR+V group; control) at 9 and 15 months of age. The control regimen largely reflects the optimum standard of care available in India under the IAP recommendations. ${ }^{18}$

Among the six centres, the centre in Bangalore did not enrol participants according to the randomisation scheme and enrolment ceased at a small number of participants because the investigator was transferred (see online supplementary table). Participants were excluded from the study if they had received any investigational $\mathrm{drug} /$ vaccine 30 days before the study vaccine or immunosuppressants/immune-modifying drugs/blood products 6 months before the study. Participants previously vaccinated against/exposed to measles, mumps, rubella and varicella or with a history of these diseases could not participate. A history of allergy likely to be aggravated by any of the vaccine components, neurological disease/seizures, chronic illness or family history of immunodeficiency, or symptoms of acute illness at the time of enrolment were other reasons for exclusion. Vaccination was postponed for participants with a rectal 
temperature $\geq 38.0^{\circ} \mathrm{C} /$ an axillary temperature $\geq 37.5^{\circ} \mathrm{C}$. Participants were also excluded if they lived in a household with newborn infants or pregnant women who have not contracted chickenpox previously or immunocompromised individuals.

The study adhered to Good Clinical Practice, the Declaration of Helsinki and all applicable regulations. The participating centres' Institutional Ethics Committees/Institutional Review Boards ${ }^{31}$ reviewed and approved the protocol. Parents/guardians provided written informed consent before performing any studyrelated procedures.

\section{Study vaccines}

All study vaccines: MMR (Priorix), varicella (Varilrix) and MMRV (Vammrix (same as Priorix-Tetra)) were manufactured by GSK, Belgium. The minimum expected potencies for measles, rubella and varicella were identical between the $\mathrm{MMR}+\mathrm{V}$ and MMRV vaccines. ${ }^{30}$ The minimum expected potency for the mumps content was higher in the MMRV vaccine $\left(\geq 10^{4.4}\right.$ median cell culture infective dose $\left.\left(\mathrm{CCID}_{50}\right)\right)$ than in the MMR vaccine $\left(\geq 10^{3.7} \operatorname{CCID}_{50}\right)$. The vaccines supplied in monodose vials contained a freeze-dried pellet which was reconstituted with the diluent (provided in a prefilled syringe) before a subcutaneous injection into the anterolateral thigh.

\section{Immunogenicity assessment}

Blood samples were collected at prevaccination and 43 days after doses 1 and 2. Antibody titres were measured using a commercial ELISA (Enzygnost, Dade Behring, Marburg, Germany) with cut-off values of $150 \mathrm{mIU} / \mathrm{mL}$ (measles), $231 \mathrm{U} / \mathrm{mL}$ (mumps) and $4 \mathrm{IU} /$ $\mathrm{mL}$ (rubella). For varicella, antibody titres were measured using an immunofluorescence assay (Virgo, Hemagen Diagnostics, Columbia, Maryland, USA; assay cut-off value of 4 /dilution).

\section{Reactogenicity and safety assessment}

Parents/guardians used diary cards to record the occurrence of solicited local symptoms (pain, redness and swelling at the injection site) for 4 days after each dose and solicited general symptoms (fever (axillary temperature $\geq 37.5^{\circ} \mathrm{C} /$ rectal temperature $\geq 38^{\circ} \mathrm{C}$ ), rash/exanthema, parotid/salivary gland swelling and any suspected signs of meningeal irritation, including febrile convulsions) for 43 days after each dose. Body temperature was measured daily via the rectal/axillary route for 15 days after each vaccination. Between days 15 and 42, the presence of fever was monitored using a temperaturesensitive pad, ${ }^{32}$ and if fever was suspected, the temperature was accurately measured with a thermometer. There were two follow-up visits with the investigator at each study centre, one visit at 42-56 days following each vaccine administration. During these visits, diary cards were returned to the investigator for assessment.
Unsolicited symptoms were recorded for 43 days after each dose, and the occurrence of serious adverse events (SAEs) was recorded throughout the study. The intensity of symptoms was graded on a scale of $0-3$. Grade 3 solicited symptoms were defined as: pain: the child cried when the limb was moved or a spontaneously painful limb; redness and swelling: injection site surface diameter $>20 \mathrm{~mm}$; fever: axillary temperature $>39^{\circ} \mathrm{C} /$ rectal temperature $>39.5^{\circ} \mathrm{C}$. Unsolicited symptoms (including SAEs) were defined as grade 3 when they prevented normal daily activity.

\section{Statistical analyses}

All statistical analyses were performed using SAS V.9.2, and $95 \%$ CIs were calculated using Proc StatXact V.8.1. The sample size was estimated taking into consideration the co-primary objectives of non-inferiority. Noninferiority was achieved if the lower limit of the twosided standardised asymptotic $95 \%$ CI for the difference in seroconversion rates between the two treatment groups and control group (MMRV/MMRV-MMR/MMR $+\mathrm{V}$; MMR/MMRV-MMR/MMR+V) was $\geq-10 \%$ for each vaccine antigen, 43 days postdose 2 . Similarly, the secondary non-inferiority objective was achieved if the lower limit of the two-sided standardised asymptotic $95 \%$ CI for the difference in seroconversion rates between the MMRV/MMRV group and pooled MMR results from the MMR/MMRV and MMR/MMR+V groups, 43 days postdose 1 was $\geq-10 \%$ for measles, mumps and rubella. Considering that up to $25 \%$ of the participants enrolled could be non-evaluable, a total of 450 participants (180 participants in each of the treatment groups and 90 participants in the control group) were to be enrolled in the study. A sample size of 130 evaluable participants in each treatment group and 65 evaluable participants in the control group was planned, which gave a power of at least $93.91 \%$ with a non-inferiority margin of $10 \%$ for all antigens to meet the co-primary objectives. A central randomisation system using a minimisation algorithm provided each child with a unique treatment number. A randomisation (2:2:1) blocking scheme ensured that the balance between treatments was maintained by providing a unique treatment number that identified the vaccine dose to be administered to the participants. Furthermore, given the different physical characteristics of the study vaccines and the number of injections between study groups, the study was conducted in an open manner wherein the treatment allocation of participants was known to the investigators and the parents/ guardians.

Immunogenicity analysis was performed on the according-to-protocol (ATP) cohort which included all participants for whom prevaccination and postvaccination serology results were available and who complied with study procedures. Seroconversion rates (defined as the appearance of antibodies (ie, antibody concentration/titre $\geq$ cut-off value) in the serum of participants who were seronegative before vaccination), and 
geometric mean titres (GMTs) were calculated with exact $95 \%$ CIs for antibodies against each vaccine antigen after each dose. The 95\% CIs for the GMTs were obtained by exponential transformation of the $95 \%$ CI for the mean of the log-transformed titre.

Safety analysis was performed on the total vaccinated cohort (TVC) which included all vaccinated participants. Solicited and unsolicited symptoms reported for the participants during the respective postvaccination periods were calculated with exact 95\% CIs. All SAEs reported during the entire conduct of the study were described.

\section{RESULTS}

\section{Demographics}

All 450 participants enrolled in the study were vaccinated and included in the TVC: MMRV/MMRV $(\mathrm{n}=180) ; \quad$ MMR/MMRV ( $\mathrm{n}=180)$ and $\mathrm{MMR} / \mathrm{MMR}+\mathrm{V}$ $(n=90)$. Of these, 382 were included in the ATP cohort for immunogenicity: MMRV/MMRV ( $\mathrm{n}=151)$; MMR/ MMRV ( $\mathrm{n}=156)$ and MMR/MMR+V ( $\mathrm{n}=75$; figure 1$)$. The median age of participants in the ATP was 9 months (range 9-10 months); $51.7 \%$ were male and all participants were Indian. No demographic variations were observed between the study groups (see online supplementary table).

\section{Immunogenicity}

The proportion of initially seropositive participants for measles, mumps and rubella was $<2.7 \%$ in all three groups. For varicella, $7.4 \%$ participants in the MMRV/ MMRV group, $8.3 \%$ in the MMR/MMRV group and $2.7 \%$ in the MMR/MMR+V group were initially seropositive. After dose 1 , the seroconversion rates ranged from $87.5 \%$ to $93.2 \%$ for measles, $83.3 \%$ to $86.1 \%$ for mumps and $98.7 \%$ to $100 \%$ for rubella (table 1). Postdose 2, seroconversion rates were $100 \%$ for measles, mumps and rubella and at least $95.8 \%$ for varicella. Across the three vaccination groups, the observed GMTs to measles, mumps and rubella increased between doses 1 and 2 (table 1). The co-primary objectives of noninferiority with respect to seroconversion rates 43 days after dose 2 were achieved for all vaccine antigens, that is, the lower bound of the $95 \%$ CIs for the difference in seroconversion rates between groups (MMRV/MMRV vs $\mathrm{MMR} / \mathrm{MMR}+\mathrm{V} ; \mathrm{MMR} / \mathrm{MMRV}$ vs $\mathrm{MMR} / \mathrm{MMR}+\mathrm{V})$ was $\geq-10 \%$.

The secondary objective of non-inferiority of the MMRV/MMRV group and pooled MMR results from the $\mathrm{MMR} / \mathrm{MMRV}$ and MMR/MMR+V groups in terms of seroconversion rates 43 days postdose 1 for measles, mumps and rubella was also achieved (table 2).

\section{Reactogenicity and safety}

During the 43-day postvaccination period, the occurrence of solicited and unsolicited symptoms ranged between $51.1 \%$ and $56.1 \%$ after dose 1 and $36.2 \%$ and $37.3 \%$ after dose 2 across the three vaccine groups. During the 4-day postdose 1 follow-up period, injection site pain was the most commonly reported solicited local

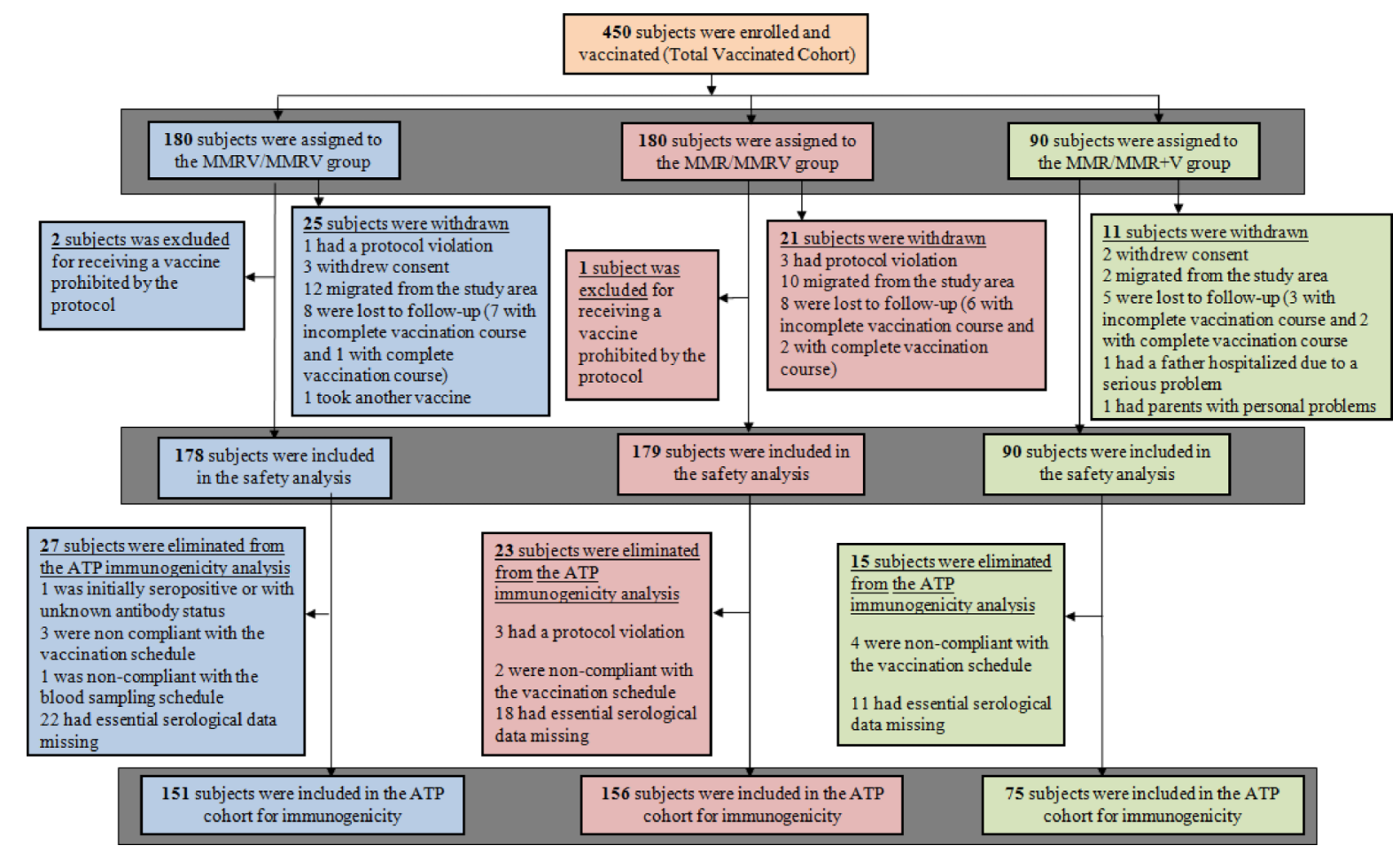

Figure 1 Participant disposition (ATP, according-to-protocol; MMR, measles-mumps-rubella vaccine; MMRV, measles-mumps-rubella-varicella vaccine; MMR+V, MMR+varicella vaccine). 


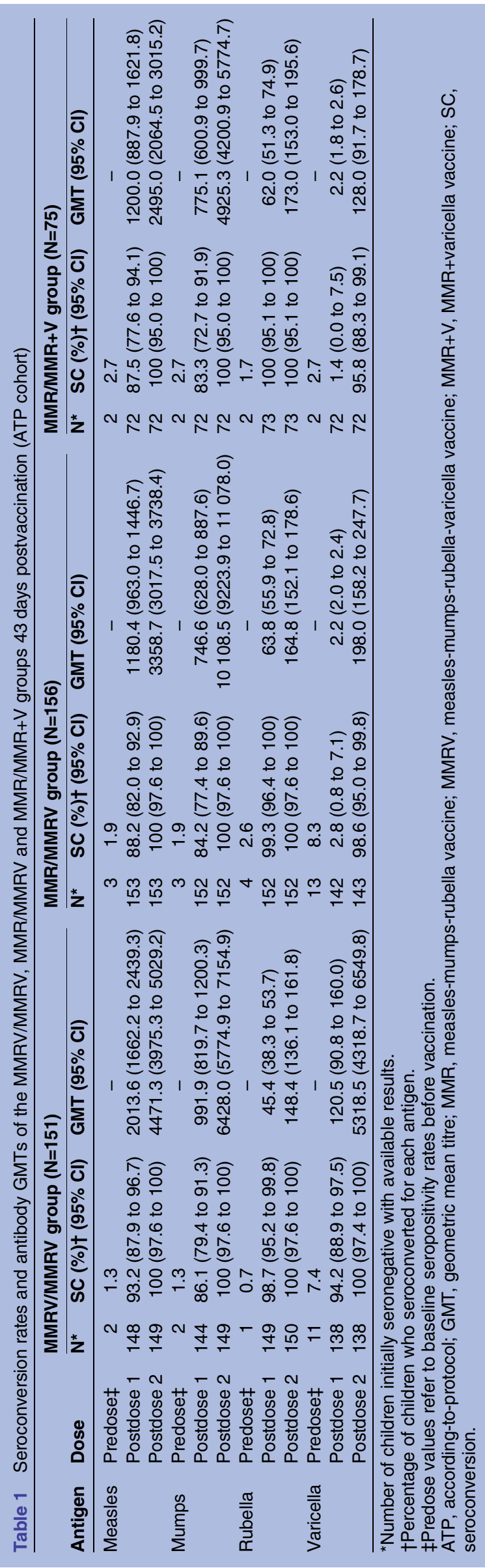

symptom: MMRV/MMRV (11.5\%), MMR/MMRV (7\%) and MMR/MMR+V (10.7\%). Postdose 2, injection site symptoms were reported by fewer than $6.5 \%$ of participants (table 3 ). Redness was the grade 3 local symptom reported by three participants $(1.9 \%)$ in the MMRV/ MMRV group, postdose 2.

Fever was the most commonly reported solicited general symptom in all three vaccine groups during the 43-day postvaccination follow-up period after each dose (table 3). The observed incidence of fever across all vaccine groups decreased between doses 1 and 2 during the 15-day and 43-day postvaccination follow-up periods (table 3).

An absence of a clear peak in the prevalence of fever during the 43-day period after dose 1 for all three vaccine groups is depicted in figure 2A. Postdose 2 fever is depicted in figure 2B. Rash occurred in one participant after the first dose of MMRV in the MMRV/MMRV group as compared with three participants after the first MMR dose (MMR/MMRV (n=2); MMR/MMR+V (n=1) groups). Only one participant developed rash after dose 2 in the MMR/MMRV group. There were no reports of meningeal irritation, febrile convulsions or parotid gland swelling during the 43-day period after each vaccine dose.

At least one unsolicited symptom was reported in $20.6 \%$ of participants after the first dose of MMRV in the MMRV/MMRV group and in $21.7 \%$ and $20.0 \%$ of participants after the first dose of MMR in the MMR/ MMRV and MMR/MMR+V groups, respectively. The most commonly reported symptoms in each group were: upper respiratory tract infection in the MMRV/MMRV group $(\mathrm{n}=10 ; 5.6 \%)$; cough in the MMR/MMRV group $(\mathrm{n}=10 ; 5.6 \%)$; and nasopharyngitis, rhinitis and cough in the MMR/MMR+V group $(n=6 ; 6.7 \%$ for each symptom). Similarly, at least one unsolicited symptom was reported in $10.6 \%$ of participants after the second dose of MMRV in the MMRV/MMRV group, in $10.0 \%$ of participants who received their first dose of MMRV in the MMR/MMRV groups and in $12.2 \%$ of participants following dose 2 of $\mathrm{MMR}+\mathrm{V}$ in the $\mathrm{MMR} / \mathrm{MMR}+\mathrm{V}$ group. The most commonly reported symptoms in each group were: rhinitis and cough in the MMRV/MMRV group ( $n=6 ; 3.3 \%$ for each symptom) and rhinitis in the MMR/MMRV $(\mathrm{n}=7 ; 3.9 \%)$ and MMR/MMR+V groups $(\mathrm{n}=5 ; 5.6 \%)$.

Overall, 18 SAEs occurred in the study. At least one SAE occurred in 13 participants (MMRV/MMRV $(n=7)$; MMR/MMRV ( $n=6) ;$ MMR/MMR+V ( $=0)$ groups). The most commonly reported SAEs were lower respiratory tract infection (in two participants) in the MMRV/ MMRV group, and gastroenteritis (in three participants) in the MMR/MMRV group. Other SAEs reported were gastroenteritis, pneumonitis, wheezing, viral infection, pneumonia, febrile convulsion, upper respiratory tract inflammation, dehydration and bronchiolitis. All SAEs resolved without sequelae and were considered unrelated to vaccination. 
Table 2 Postdose 1 seroconversion rates between the MMRV/MMRV group and pooled MMR/MMRV+MMR/MMR+V groups (ATP cohort)

\begin{tabular}{|c|c|c|c|c|c|}
\hline \multirow[b]{2}{*}{ Antibody } & \multicolumn{2}{|c|}{$\begin{array}{l}\text { MMRV } \\
\text { (MMRV/MMRV group) }\end{array}$} & \multicolumn{2}{|c|}{$\begin{array}{l}\text { MMR } \\
\text { (Pooled (MMR/MMRV)+ } \\
\text { (MMR/MMR+V) groups) }\end{array}$} & \multirow{2}{*}{$\begin{array}{l}\text { Difference in percentage } \\
\text { (MMRV-MMR) } \\
\text { Per cent }(95 \% \mathrm{Cl})\end{array}$} \\
\hline & $\overline{\mathbf{N}^{*}}$ & Per cent $†$ & $\overline{\mathbf{N}^{*}}$ & Per cent $†$ & \\
\hline Measles & 148 & 93.2 & 225 & 88.0 & $5.24(-1.06$ to 11.13$)$ \\
\hline Mumps & 144 & 86.1 & 224 & 83.9 & $2.18(-5.66$ to 9.42$)$ \\
\hline Rubella & 149 & 98.7 & 225 & 99.6 & $-0.90(-4.36$ to 1.29$)$ \\
\hline Varicella & 138 & 94.2 & - & & - \\
\hline
\end{tabular}

\section{DISCUSSION}

This non-inferiority study evaluated the immunogenicity and safety of two vaccination regimens: (1) two-dose
MMRV/MMRV, (2) MMR followed by MMRV compared with a control group (MMR followed by MMR+V) when administered to healthy Indian children at 9 and

Table 3 Incidence of solicited local symptoms (during the 4-day) and fever (during the 15-day and 43-day) postvaccination period (total vaccinated cohort)

\begin{tabular}{|c|c|c|c|c|c|c|}
\hline & \multicolumn{2}{|c|}{$\begin{array}{l}\text { MMRV/MMRV group } \\
\text { Dose } 1 \mathrm{~N}^{*}=174 ; \\
\text { Dose } 2 \mathrm{~N}^{*}=155\end{array}$} & \multicolumn{2}{|c|}{$\begin{array}{l}\text { MMR/MMRV group } \\
\text { Dose } 1 \mathrm{~N}^{*}=172 \\
\text { Dose } 2 \mathrm{~N}^{*}=159\end{array}$} & \multicolumn{2}{|c|}{$\begin{array}{l}\text { MMR/MMR+V group } \\
\text { Dose } 1 N^{*}=84 \\
\text { Dose } 2 N^{*}=79\end{array}$} \\
\hline & Per cent† & $(95 \% \mathrm{Cl})$ & Per cent† & $(95 \% \mathrm{Cl})$ & Per cent† & $(95 \% \mathrm{Cl})$ \\
\hline \multicolumn{7}{|l|}{ Pain } \\
\hline Postdose 1 & 11.5 & (7.2 to 17.2$)$ & 7.0 & (3.7 to 11.9 ) & 10.7 & (5.0 to 19.4$)$ \\
\hline Postdose 2 & 5.8 & (2.7 to 10.7$)$ & 6.3 & (3.1 to 11.3 ) & 3.8 & (0.8 to 10.7$)$ \\
\hline \multicolumn{7}{|l|}{ Redness } \\
\hline Postdose 1 & 8.6 & (4.9 to 13.8$)$ & 4.7 & (2.0 to 9.0$)$ & 3.6 & $(0.7$ to 10.1$)$ \\
\hline Postdose 2 & 6.5 & (3.1 to 11.5 ) & 3.8 & (1.4 to 8.0$)$ & 0.0 & (0.0 to 4.6$)$ \\
\hline \multicolumn{7}{|l|}{ Swelling } \\
\hline Postdose 1 & 4.6 & (2.0 to 8.9$)$ & 2.9 & (1.0 to 6.7$)$ & 3.6 & (0.7 to 10.1$)$ \\
\hline Postdose 2 & 5.8 & (2.7 to 10.7 ) & 3.8 & (1.4 to 8.0$)$ & 0.0 & (0.0 to 4.6$)$ \\
\hline \multicolumn{7}{|c|}{ Fever (15 days postdose 1 ) } \\
\hline Any & 32.2 & (25.3 to 39.7$)$ & 28.5 & (21.9 to 35.9 ) & 21.7 & (13.4 to 32.1$)$ \\
\hline Grade $3\left(>39.5^{\circ} \mathrm{C}\right)$ & 3.4 & $(1.3$ to 7.4$)$ & 1.7 & $(0.4$ to 5.0$)$ & 1.2 & $(0.0$ to 6.5$)$ \\
\hline Related & 28.2 & $(21.6$ to 35.5$)$ & 24.4 & (18.2 to 31.5$)$ & 16.9 & (9.5 to 26.7 ) \\
\hline Medical advice & 6.3 & (3.2 to 11.0$)$ & 7.6 & (4.1 to 12.6$)$ & 2.4 & (0.3 to 8.4$)$ \\
\hline \multicolumn{7}{|c|}{ Fever (15 days postdose 2 ) } \\
\hline Any & 17.4 & (11.8 to 24.3$)$ & 13.2 & (8.4 to 19.5$)$ & 15.2 & (8.1 to 25.0$)$ \\
\hline Grade $3\left(>39.5^{\circ} \mathrm{C}\right)$ & 1.3 & $(0.2$ to 4.6$)$ & 1.3 & $(0.2$ to 4.5$)$ & 0.0 & (0.0 to 4.6$)$ \\
\hline Related & 13.5 & (8.6 to 20.0$)$ & 11.9 & (7.4 to 18.0$)$ & 12.7 & (6.2 to 22.0$)$ \\
\hline Medical advice & 3.9 & (1.4 to 8.2$)$ & 1.3 & $(0.2$ to 4.5$)$ & 1.3 & (0.0 to 6.9$)$ \\
\hline \multicolumn{7}{|c|}{ Fever (43 days postdose 1 ) } \\
\hline Any & 43.7 & (36.2 to 51.4$)$ & 40.7 & (33.3 to 48.4$)$ & 32.5 & (22.6 to 43.7 ) \\
\hline Grade $3\left(>39.5^{\circ} \mathrm{C}\right)$ & 6.3 & (3.2 to 11.0$)$ & 2.9 & $(1.0$ to 6.7$)$ & 1.2 & $(0.0$ to 6.5$)$ \\
\hline Related & 30.5 & (23.7 to 37.9$)$ & 27.9 & (21.3 to 35.2$)$ & 18.1 & (10.5 to 28.0$)$ \\
\hline Medical advice & 13.8 & (9.0 to 19.8$)$ & 16.9 & (11.6 to 23.3 ) & 4.8 & (1.3 to 11.9$)$ \\
\hline \multicolumn{7}{|c|}{ Fever (43 days postdose 2) } \\
\hline Any & 26.5 & (19.7 to 34.1$)$ & 23.3 & (16.9 to 30.6$)$ & 27.8 & (18.3 to 39.1$)$ \\
\hline Grade $3\left(>39.5^{\circ} \mathrm{C}\right)$ & 1.3 & $(0.2$ to 4.6$)$ & 3.8 & $(1.4$ to 8.0$)$ & 2.5 & $(0.3$ to 8.8$)$ \\
\hline Related & 14.2 & (9.1 to 20.7 ) & 13.2 & (8.4 to 19.5$)$ & 12.7 & (6.2 to 22.0$)$ \\
\hline Medical advice & 4.5 & (1.8 to 9.1$)$ & 5.6 & (2.6 to 10.5$)$ & 7.6 & (2.8 to 15.8$)$ \\
\hline
\end{tabular}

${ }^{*}$ Number of children with at least one documented dose.

†Percentage of children reporting the symptom at least once.

MMR, measles-mumps-rubella vaccine; MMRV, measles-mumps-rubella-varicella vaccine; MMR+V, MMR+varicella vaccine. 

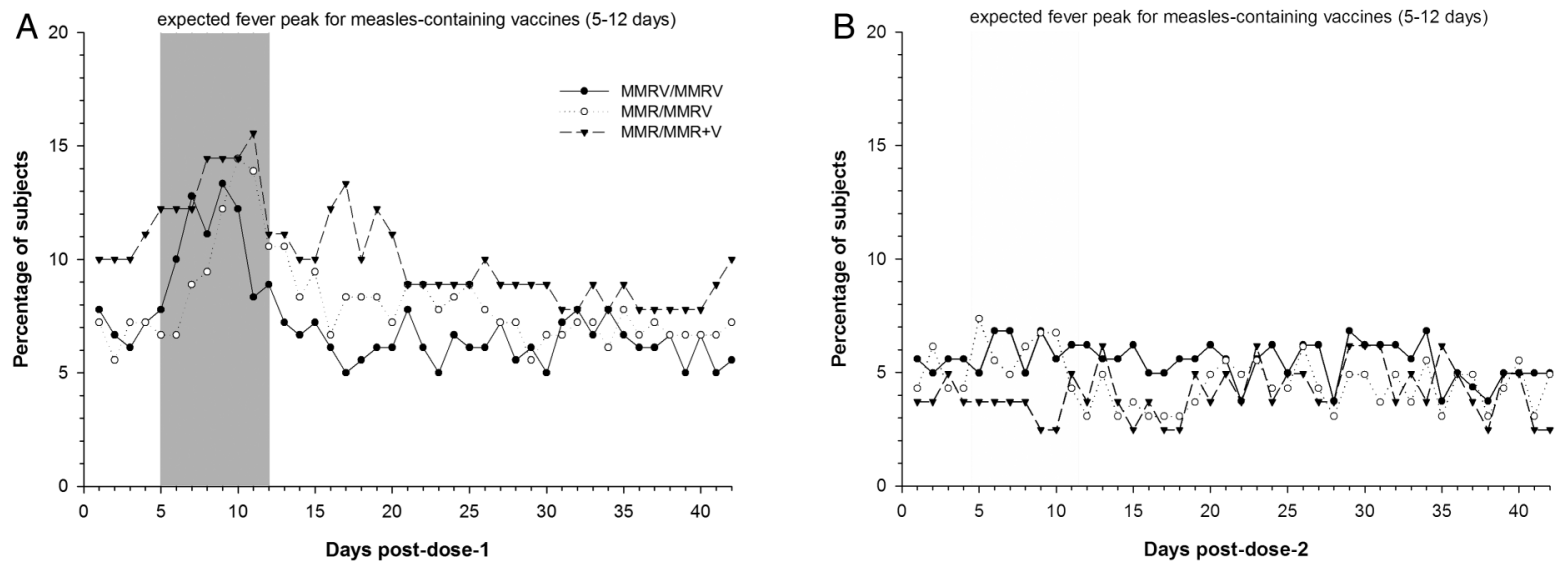

Figure 2 (A) Prevalence of fever during the 43-day postvaccination period after dose 1 (total vaccinated cohort). (B) Prevalence of fever during the 43-day postvaccination period after dose 2 (total vaccinated cohort). MMR, measles-mumps-rubella vaccine;

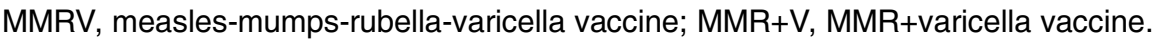

15 months of age. The co-primary non-inferiority criterion ruling out a $10 \%$ difference in seroconversion rates postdose 2 of MMRV/MMRV and MMR/MMRV compared with $\mathrm{MMR} / \mathrm{MMR}+\mathrm{V}$ was achieved for all antigens, indicating that the immune responses elicited by the two vaccination regimens were non-inferior to those elicited by the control regimen. Although the two-dose MMRV/ MMRV schedule is not included in several immunisation programmes, this regimen has been established to be non-inferior to the two-dose MMR+V schedule in separate studies in Germany and Singapore. ${ }^{30} 33$ Additionally, on comparing the postdose 1 responses, one dose of MMRV in the MMRV/MMRV group elicited noninferior immune responses against measles, mumps and rubella compared with pooled results of one dose of $\mathrm{MMR}$ in the MMR/MMRV and MMR/MMR+V groups.

In the three vaccine groups, we observed low baseline seropositivity rates $(<2.7 \%)$ for measles, mumps and rubella in participants at 9 months of age. This finding suggests a possible decline in circulating maternal antibodies and measles infection by this age which would support 9 months as a suitable age for initial vaccination. However, it should be noted that this finding is inconsistent with a notable Indian study conducted approximately a decade ago that suggested the persistence of high circulating maternal antibodies at 9 months of age with baseline seropositivity rates of $15 \%$ for measles and $20 \%$ each for mumps and rubella. ${ }^{11}$ Additionally, while lowering the measles vaccination age in low-income countries is supported by many, vaccinating at 9 months or earlier may mean that the immune system has not reached optimum maturity to mount an effective response and provide effective long-term protection against measles or the other diseases with just a single vaccine dose. ${ }^{34} 35$ The seroconversion rates for all antigens in the MMRV/MMRV group were consistent with previous observations in Singaporean children at 9 months of age by Goh et al. ${ }^{33}$ However, with a first dose of MMR, the observed postdose 1 seroconversion rates to measles (MMR/MMRV $=88.2 \%$; $\mathrm{MMR} / \mathrm{MMR}$
$+\mathrm{V}=87.5 \%)$ and mumps (MMR/MMRV $=84.2 \%$; MMR/ $\mathrm{MMR}+\mathrm{V}=83.3 \%)$ in this study were somewhat lower than those reported by Schuster et $a l^{30}{ }^{30}$ following administration of a first dose of MMR to children aged 11-21 months in Germany (measles 93.4\%; mumps 93.6\%). A contrast in immune responses between the current study and the German study may be attributed to the age at vaccination, maturity of the immune system and circulating maternal antibodies. Also, the higher GMTs observed in measles in the MMRV/MMRV group compared with the MMR/MMRV and MMR/MMR+V groups could translate to more effective protection in a highly endemic measles environment where coverage from a second dose of a measles-containing vaccine remains variable throughout the country. ${ }^{6}$ Lower seroconversion rates with other live-attenuated vaccines (such as the oral polio vaccine and the rotavirus vaccine) have also been observed in India and South Asia compared with more industrialised settings. ${ }^{36-38}$

Nevertheless, we observed high seroconversion rates for all vaccine antigens following the administration of a second vaccination at 15 months of age, indicating that an early two-dose immunisation strategy when the first dose is administered as early as 9 months elicits a satisfactory immune response. An interesting observation was the markedly high GMT against mumps postdose 2 in the MMR/MMRV (10 108.5) group compared with the MMR/MMR+V (4925.3) and MMRV/MMRV (6428.0) groups. This observation has not been reported previously despite the higher mumps antigen content in the MMRV vaccine when compared with the MMR vaccine and studies that evaluate this finding may be needed in the future.

Early administrations of all three vaccination regimens were well tolerated when administered to young children at 9 and 15 months of age. Similar differences in solicited general symptoms have been observed in studies conducted in the Indian subcontinent with other live-attenuated viral vaccines (such as Rotarix) compared with other countries. ${ }^{37} 39$ Notably, unlike previous 
studies conducted in other countries, ${ }^{28-30} 33$ this study did not demonstrate any difference in fever rates between MMRV and MMR when used as a first dose of measles-containing vaccine. Although this may be related to the epidemiological context in India, which differs from developed countries, the reason is unclear. In general, the reporting rate of fever was also lower than that seen in other studies. ${ }^{28-30} 33$ Again, this may be due to the younger age of children enrolled, or the presence of maternal antibodies, which may have limited measles virus replication postdose 1 , resulting in the blunting of immune response and fever response rates; or cross-cultural/geographical differences in the reporting of symptoms. Further data may be needed to determine if there is in fact a difference in the reactogenicity profile between developed and developing countries.

It is possible to eliminate measles from a specific region by sustaining high immunisation coverage as is evident from Latin America, Finland and the USA; ${ }^{40-42}$ countries such as Finland have also successfully eliminated mumps and rubella using the MMR vaccine. ${ }^{41}$ In view of the ongoing transmission and high mortality risk of measles in India, immunisation coverage of $\geq 95 \%$ for the first and second doses would be required to ensure prevention of measles virus transmission. ${ }^{43}$ Currently, the Indian national immunisation schedule's 9 months single dose immunisation coverage is $74 \% ;{ }^{44}$ however, as this represents the average, coverage rates in some parts of the country may be even lower. Several European countries, where the first MMR dose is administered to children in the second year of life (12-24 months), continue to face relatively low immunisation coverage rates resulting in measles and mumps outbreaks, ${ }^{24}$ as was recently observed even in South Wales. ${ }^{10}$ An earlier vaccination schedule was implemented in Germany as a result of such outbreaks, whereby the first dose is now administered at 11-14 months followed by the second dose at $15-23$ months of age. ${ }^{24}$ This revised strategy achieved well-documented successful immunisation coverage rates, ${ }^{24}$ and highlights the importance of early vaccination with increased compliance and subsequently higher coverage rates.

There are a few limitations of the study that should be considered: (1) the six tertiary care centres where the study was conducted are not representative of the entire Indian population, (2) investigator bias while reporting AEs due to the open design of the study, and (3) there were no adjustments made for confounding factors (eg, centres) in the analysis.

\section{CONCLUSION}

This study demonstrates that in an Indian setting, the two-dose vaccination regimens of $\mathrm{MMRV} / \mathrm{MMRV}$ and MMR/MMRV are non-inferior to the control MMR/ $\mathrm{MMR}+\mathrm{V}$ regimen (ie, the local standard of care recommended by the IAP) ${ }^{17}$ in terms of immunological response. Both vaccination schedules demonstrated an acceptable safety profile when administered to healthy Indian children at 9 and 15 months of age. Introduction of the MMRV vaccine may facilitate effective population protection against measles, as well as against three other common childhood viral infectious diseases: mumps, rubella and varicella.

Author affiliations

${ }^{1}$ Department of Pediatrics, Bharati Vidyapeeth Deemed University, Pune, Maharashtra, India

${ }^{2}$ Department of Pediatrics, Medical College Kolkata, Kolkata, West Bengal, India

${ }^{3}$ Kanchi Kamakoti Childs Trust Hospital, Chennai, Tamil Nadu, India

${ }^{4}$ Department of Pediatrics, KEM Hospital, Pune, Maharashtra, India

${ }^{5}$ GSK Pharmaceuticals Ltd., Mumbai, Maharashtra, India

${ }^{6}$ GSK Vaccines, Wavre, Belgium

${ }^{7}$ GSK Vaccines, King of Prussia, Philadelphia, USA

Acknowledgements The authors thank the study nurses and staff members involved, and the parents and children who participated in the study. The authors acknowledge Dr Sonali Palkar for her support in conducting the clinical trial and Dipti Phatarpekar for coordinating and supervising data collection at two study sites. The authors also thank Ashmita Ravishankar for medical writing and publication coordination (GSK group of companies Vaccines), and Jarno Jansen (Keyrus Biopharma for GSK group of companies Vaccines), Geetha Subramanyam (GSK group of companies Vaccines) and Shruti MP (GSK group of companies Vaccines) for editorial assistance and publication coordination.

Contributors SL, SB, SC and $A B$ were involved in the recruitment of participants. SL critically reviewed the protocol and study report, and also provided scientific and medical inputs during the development of the study report. SB was involved in providing scientific and medical inputs to the protocol and critically reviewing the protocol. SC coordinated and supervised data collection at one site and carried out the initial analyses. $A B$ provided critical inputs to the study report and reviewed the content of the study report. SM and SD conceptualised and designed the study. SM also supervised data collection at all sites. MP was involved in data analysis and interpretation. SD and $\mathrm{OH}$ provided input to the interpretation of clinical data. All authors critically reviewed and revised the manuscript during the development and approved the final version of the manuscript. All authors are guarantors and accept full responsibility for the work and/or conduct of the study, had access to the data, and controlled the decision to publish.

Funding This study was sponsored and funded by GlaxoSmithKline Biologicals SA, which was involved in all stages of the study conduct and analysis and also took charge of all costs associated with the development and the publishing of the manuscript.

Competing interests SL declares to have received an institutional grant (for BVDU Medical college, Department of Pediatrics) and travel support for the meeting from the GSK group of companies. He also declares to have received fees for lecture at Pedicon 2011. SB declares that The childs trust medical research foundation received a grant from the GSK group of companies. $A B$ declares to have received an institutional grant for conducting this trial and has pending institutional grants from the GSK group of companies for other vaccine trials. He also declares to have received support for travel/ accommodation while participating in investigator meetings and remuneration for participating in a meeting on adjuvants from the GSK group of companies. $\mathrm{SM}, \mathrm{SD}, \mathrm{MP}$ and $\mathrm{OH}$ are employees of the GSK group of companies; SD and $\mathrm{OH}$ declare to have stock options. MP declares that he received payment/fees for review of statistical analysis and for reviewing this manuscript from the GSK group of companies.

\section{Patient consent Obtained.}

Ethics approval Institutional Ethics Committees/Institutional Review Boards of each participating centre. 
Provenance and peer review Not commissioned; externally peer reviewed.

Data sharing statement On authorisation or termination of development of this medicine, anonymised patient-level data underlying this study will be made available to independent researchers, subject to review by an independent panel, at http://www.clinicalstudydatarequest.com.

Open Access This is an Open Access article distributed in accordance with the Creative Commons Attribution Non Commercial (CC BY-NC 4.0) license, which permits others to distribute, remix, adapt, build upon this work noncommercially, and license their derivative works on different terms, provided the original work is properly cited and the use is non-commercial. See: http:// creativecommons.org/licenses/by-nc/4.0/

\section{REFERENCES}

1. World Health Organization. Measles vaccines: WHO position paper. Wkly Epidemiol Rec 2009;84:349-60.

2. World Health Organization. Mumps virus vaccines. Wkly Epidemiol Rec 2007;82:49-60.

3. World Health Organization. Rubella vaccines: WHO position paper. Wkly Epidemiol Rec 2011;86:301-16.

4. Marin M, Güris D, Chaves SS, et al; Advisory Committee on Immunization Practices, Centers for Disease Control and Prevention (CDC). Prevention of varicella: recommendations of the Advisory Committee on Immunization Practices (ACIP). MMWR Recomm Rep 2007:56(RR04): 1-40.

5. Simons E, Ferrari M, Fricks J, et al. Assessment of the 2010 global measles mortality reduction goal: results from a model of surveillance data. Lancet 2012:379:2173-8.

6. World Health Organization Media Centre. WHO: measles deaths decline, but elimination progress stalls in some regions. 2013. http:// www.who.int/mediacentre/news/notes/2013/measles_20130117/en/ (Accessed 25 Nov 2013).

7. Centers for Disease Control and Prevention (CDC). MeaslesUnited States, January 1-August 24, 2013. MMWR Morb Mortal Wkly Rep 2013;62:741-3.

8. European Centre for Disease Prevention and Control. Monitoring current health threats, week 39/2013: measles and possible polio re-emergence in focus. http://ecdc.europa.eu/en/press/news/ layouts/forms/News_DispForm.aspx?List=8db7286c\%2Dfe2d\% 2D476c\%2D9133\%2D18ff4cb1b568\&ID=872\&RootFolder=\%2Fen\% 2Fpress\%2Fnews\%2FLists\%2FNews\&Web=86661a14\%2Dfb61\% 2D43e0\%2D9663\%2D0d514841605d (accessed 20 Apr 2015)

9. Wise J. Measles outbreak hits northeast England. BMJ 2013;346: f662.

10. Wise J. Largest group of children affected by measles outbreak in Wales is 10-18year olds. BMJ 2013;346:f2545.

11. Yadav S, Thukral R, Chakarvarti A. Comparative evaluation of measles, mumps \& rubella vaccine at $9 \& 15$ months of age. Indian $J$ Med Res 2003;118:183-6.

12. Ramamurty N, Murugan S, Raja D, et al. Serosurvey of rubella in five blocks of Tamil Nadu. Indian J Med Res 2006;123:51-4.

13. Verma R, Khanna $P$, Chawla $S$. Rubella vaccine: new horizon in prevention of congenital rubella syndrome in India. Hum Vaccin Immunother 2012;8:1-3.

14. Verma R, Bairwa M, Chawla S, et al. Should the chickenpox vaccine be included in the national immunization schedule in India? Hum Vaccin 2011;7:874-7.

15. World Health Organization. Confirmed international spread of wild poliovirus from Pakistan. Wkly Epidemiol Rec 2011;86:437-44

16. Singhal T, Amdekar YK, Thacker N. IAP committee on immunization. Indian Pediatr 2007;44:390-2.

17. Verma R, Khanna P, Bairwa M, et al. Introduction of a second dose of measles in national immunization program in India: a major step towards eradication. Hum Vaccin 2011;7:1109-11.

18. Indian Academy of Pediatrics Committee on Immunization (IAPCOI). Consensus recommendations on immunization and IAP immunization timetable 2012. Indian Pediatr 2012;49:549-64.

19. Ozdemir H, Candir MO, Karbuz A, et al. Chickenpox complications, incidence and financial burden in previously healthy children and those with an underlying disease in Ankara in the pre-vaccination period. Turk J Pediatr 2011;53:614-25.

20. Bilcke J, Ogunjimi B, Marais C, et al. The health and economic burden of chickenpox and herpes zoster in Belgium. Epidemiol Infect 2012;140:2096-109.
21. Da YP, Luo LY, Song LZ. [Economic burden of inpatient of varicella in Shandong, Gansu and Hunan provinces, 2007]. Zhongguo Yi Miao He Mian Yi 2009;15:438-42.

22. Department of Health. Childhood immunizations guidance. http:// www.gov.im/lib/docs/health/Public Health/Immunisation/ imm010112immunisationbookletlress.pdf (accessed 8 Nov 2012)

23. Chiu SS, Lau YL. Review of the Varilrix varicella vaccine. Expert Rev Vaccines 2005;4:629-43.

24. Vesikari T, Sadzot-Delvaux C, Rentier B, et al. Increasing coverage and efficiency of measles, mumps, and rubella vaccine and introducing universal varicella vaccination in Europe. A role for the combined vaccine. Pediatr Infect Dis J 2007;26: 632-8.

25. Rentier B, Gershon AA. European Working Group on Varicella. Consensus: varicella vaccination of healthy children-a challenge for Europe. Pediatr Infect Dis J 2004;23:379-89.

26. Czajka H, Schuster V, Zepp F, et al. A combined measles, mumps, rubella and varicella vaccine (Priorix-Tetra ${ }^{\mathrm{TM}}$ ): immunogenicity and safety profile. Vaccine 2009;27:6504-11

27. Knuf M, Faber J, Barth I, et al. A combination vaccine against measles, mumps, rubella and varicella. Drugs Today 2008;44:279-92.

28. Nolan T, McIntyre P, Roberton D, et al. Reactogenicity and immunogenicity of a live and attenuated tetravalent measles-mumps-rubella-varicella (MMRV) vaccine. Vaccine 2002;21:281-9.

29. Knuf M, Habermehl $\mathrm{P}$, Zepp $\mathrm{F}$, et al. Immunogenicity and safety of two doses of tetravalent measles-mumps-rubella-varicella vaccine in healthy children. Pediatr Infect Dis J 2006;25:12-18.

30. Schuster V, Otto W, Maurer L, et al. Immunogenicity and safety assessments after one and two doses of a refrigerator-stable tetravalent measles-mumps-rubella-varicella vaccine in healthy children during the second year of life. Pediatr Infect Dis $J$ 2008:27:724-30.

31. Indian clinical trial registry. http://ctri.nic.in/Clinicaltrials/showallp. php? mid $1=930 \&$ EncHid=\&userName=CTRI/2009/091/000750 (accessed 3 May 2013)

32. Martyn KK, Urbano MT, Hayes JS, et al. Comparison of axillary, rectal and skin-based temperature assessment in preschoolers. Nurse Pract 1988:13:31-6.

33. Goh P, Lim FS, Han HH, et al. Safety and immunogenicity of early vaccination with two doses of tetravalent measles-mumpsrubella-varicella (MMRV) vaccine in healthy children from 9 months of age. Infection 2007;35:326-33.

34. Aaby P, Martins CL, Garly ML, et al. The optimal age of measles immunisation in low-income countries: a secondary analysis of the assumptions underlying the current policy. BMJ Open 2012;12: e000761.

35. Gomber S, Arora SK, Das S, et al. Immune response to second dose of MMR vaccine in Indian children. Indian J Med Res 2011;134:302-6.

36. Patriarca PA, Wright PF, John TJ. Factors affecting the immunogenicity of oral poliovirus vaccine in developing countries: review. Rev Infect Dis 1991;13:926-39.

37. Narang A, Bose A, Pandit AN, et al. Immunogenicity, reactogenicity and safety of human rotavirus vaccine (RIX4414) in Indian infants. Hum Vaccin 2009;5:414-19.

38. Zaman K, Sack DA, Yunus M, et al. Successful co-administration of a human rotavirus and oral poliovirus vaccines in Bangladesh infants in a 2-dose schedule at 12 and 16weeks of age. Vaccine 2009;27:1333-9.

39. Vesikari T, Karvonen A, Puustinen L, et al. Efficacy of RIX4414 live attenuated human rotavirus vaccine in Finnish infants. Pediatr Infect Dis J 2004;23:937-43.

40. Andrus JK, de Quadros CA, Solorzano CC, et al. Measles and rubella eradication in the Americas. Vaccine 2011;29S:D91-6.

41. Davidkin I, Kontio M, Paunio M, et al. MMR vaccination and disease elimination: the Finnish experience. Expert Rev Vaccines 2010:9:1045-53.

42. Mulholland EK. Measles in the United States, 2006. N Engl J Med 2006;355:440-3.

43. World Health Organization. Field guidelines for measles elimination, 2004. http://www.measlesrubellainitiative.org/mi-files/Tools/ Guidelines/WPRO/Field guidelines measles elimination.pdf (accessed 26 Sep 2012).

44. World Health Organization. Immunization profile-India. http://apps who.int/immunization monitoring/en/globalsummary/ countryprofileresult.cfm?C=ind (accessed 13 Nov 2012). 